\title{
Verticalización de molares con sistema de cantilever doble: análisis de elementos finitos
}

\section{Verticalization of molars with double cantilever system: finite element analysis}

Recibido: 2017/05/02. Aceptado: 2017/07/05. Publicado: 2017/09/01

\section{Giuliana Marquez Reyes ${ }^{1}$ \\ Cristina Oliveira ${ }^{2}$}

1 Universidad San Francisco de Quito, Colegio de Ciencias de la Salud, Escuela de Odontología, Clínica Odontológica, Campus Cumbayá, oficina CO 1 06, casilla postal 1 7-1 200-841. Quito-Ecuador.

Correo electrónico: giulianamarquezr@gmail.com

2 Universidad San Francisco de Quito, Colegio de Ciencias de la Salud, Escuela de Odontología, Clínica Odontológica, Campus Cumbayá, oficina CO 106, casilla postal 17-1200-841, Quito-Ecuador.

Correo electrónico: crisaol@yahoo.com.br 


\section{Resumen}

La verticalización de molares ha sido catalogada como un reto clínico para el ortodoncista, ya que es un tratamiento que presenta una extrema complejidad y varios efectos indeseables. Uno de dichos efectos es la extrusión del molar al enderezarlo pues con la mayoría de los sistemas empleados no se tiene un correcto control vertical. El objetivo de este estudio fue determinar el sistema de fuerzas y efectos que provoca la aplicación del cantilever doble en la verticalización de un molar ya que este sistema ha sido utilizado clínicamente con el fin de verticalizar sin provocar extrusión. Dicho objetivo se cumplió mediante un estudio de elementos finitos que consiste en la creación de estructuras virtuales que semejan exactamente las mismas características de un objeto real. Después de la realización de varias simulaciones se verificó que el cantilever doble no solamente tiene la capacidad de evitar la extrusión del molar sino también la de provocar un promedio de $1.29 \mathrm{~mm}$ de intrusión de la pieza. Asimismo, se verificó que existe grandes áreas de compresión y tensión en varias zonas del molar y del tubo.

Palabras clave: verticalización, molares, cantilever doble, intrusión, estrés

\section{Abstract}

Molar uprighting has been classified as a clinical challenge since it is a treatment that presents extreme complexity and several undesirable effects. One of these effects is the extrusion of the molar because most of the systems used do not have a correct vertical control. The objective of this study is to determine the forces and effects that cause the application of double cantilever in the molar uprighting since this system has been used clinically in the past to straighten without causing extrusion. This objective will be fulfilled through a study of finite elements that consists in the creation of virtual structures that act exactly like the real object. After performing several simulations, it was verified that the double cantilever has not only the ability to avoid molar extrusion but also to cause an average of $1.29 \mathrm{~mm}$ of molar intrusion. Also, it was verified that there are large areas of compression and tension in several zones of the molar and the tube.

Key words: uprighting, molars, double cantilever, intrusion, stress 


\section{Introducción}

Una de las maloclusiones comunes en los adultos es la mesioinclinación de molares por la pérdida prematura de piezas adyacentes. Es muy importante valorar el tiempo transcurrido desde la pérdida de la pieza, ya que el grado de inclinación que puede tener un segundo molar a los pocos meses de la pérdida del primer molar va a ser mucho menor que si la comparamos con la inclinación que se presentaría después de años de la exodoncia de la pieza adyacente. El alineamiento dental en estos casos puede facilitar la rehabilitación y el tratamiento periodontal con el fin de que el paciente tenga una buena salud oral. A pesar de ello, la verticalización de molares ha sido un reto clínico que requiere una evaluación adecuada. A lo largo de la historia se han utilizado varios sistemas biomecánicos entre los cuales están: el cantiléver, varios dobleces en arcos seccionales, microimplantes, entre otros. ${ }^{1,2,3}$

La inclinación de las piezas adyacentes al espacio puede provocar un defecto óseo vertical que resulta en bolsas periodontales infraóseas en la superficie mesial del diente inclinado. Además, se pueden presentar contactos prematuros en relación céntrica, interferencias en los movimientos excéntricos que podrían provocar recesiones gingivales, inflamación del ligamento periodontal, dolor, etc. de los dientes opuestos. Por otro lado, la rehabilitación del diente perdido es difícil en el caso de que haya mucha inclinación y problema periodontal, de manera que es necesaria la intervención del ortodoncista para la alineación correcta del sector posterior afectado. La verticalización de molares permite la salud oclusal y periodontal, la paralelización de las raíces y su correcta posición con respecto al plano oclusal y el cumplimiento de la ley de carga axial. 4

El tipo de mecánica a utilizar para la verticalización dependerá del grado de inclinación del molar afectado. Una vez evaluada la biomecánica se debe programar el tipo de anclaje que se debe usar para evitar efectos adversos; lo cual quiere decir, que se debe realizar una evaluación exhaustiva del punto donde se va a aplicar las fuerzas, dónde está ubicado el centro de resistencia y su relación con el centro de rotación, la existencia de momentos, etc. Es así que dentro de este análisis se determinará la cantidad de dientes que van a servir como anclaje. Adicional a esto, se debe analizar el tipo de movimiento que se requiere; es decir, intrusión, extrusión, distalización, mesialización. El plan de tratamiento para la verticalización depende de la cantidad de dientes ausentes, el tiempo transcurrido desde la extracción, el tipo de tejido óseo remanente, la posición y angulación de los dientes adyacentes y antagonistas al espacio libre, estado periodontal, oclusión y biotipo facial del paciente; por lo cual, el clínico debe estar atento a estos factores para que el tratamiento sea un éxito clínico. ${ }^{3}$

Cabe recalcar que la mayoría de dispositivos usados para la verticalización tienen fuerzas extrusivas para el molar a tratar, lo cual en ciertos casos puede ser un efecto perjudicial para la estética y salud oclusal del paciente. Sin embargo, se conoce que el cantilever doble confeccionado en TMA 0.017" x 0,025", con una activación tip back de $45^{\circ}$ y $30 \mathrm{~mm}$ de longitud puede evitar la extrusión del molar e inclusive provocar un movimiento de intrusión en el mismo, lo que es considerado una ventaja importante para el tratamiento. Es así que el objetivo de esta investigación pretendió determinar el sistema de fuerzas y efectos que provoca la aplicación del cantilever doble en la verticalización de un molar; específicamente, se intenta verificar si existe un movimiento de intrusión en el molar a tratar y que tipo de presión se realiza con el sistema de fuerzas en las diferentes partes del diente.

La técnica a utilizar va a ser el método de elementos finitos que puede provocar interacciones entre los diferentes elementos simulados en un programa de computación: 
Autodesk Inventor Professional 3D, con el fin de evaluar fuerzas, movimientos y efectos que dichas interacciones pueden provocar en uno y otro elemento. Dentro de la especialidad de ortodoncia se usa para entender los efectos de las fuerzas aplicadas en distintos sistemas biomecánicos y los movimientos que éstas pueden provocar de acuerdo a los tres ejes del espacio: $x, y, z$. Dicha evaluación permite determinar también las consecuencias del estrés y tensión en partes del cuerpo que son difíciles de acceder sin causar daño, como por ejemplo el ligamento periodontal y las superficies del hueso alveolar cercanas al diente. ${ }^{\mathbf{5}}$

\section{Materiales y Métodos}

La presente investigación se define como un estudio in vitro ya que su muestra no comprende organismos vivos. Asimismo, es un estudio observacional y cualitativo ya que mediante un software se pretende observar y describir los efectos de las fuerzas que puede provocar un cantilever doble al verticalizar un molar. Por último, es un estudio cuantitativo pues se busca medir la cantidad de movimiento de intrusión, si este existiese, que provocaría el resorte cantilever doble sobre el molar verticalizado. El estudio se basa en una muestra virtual de la arcada mandibular que represente la mesioinclinación de un segundo molar por

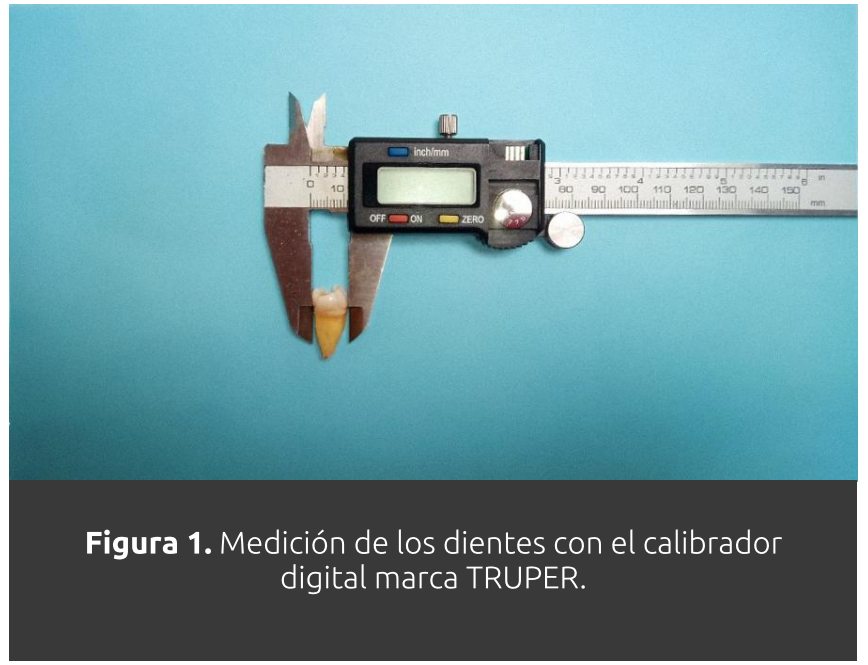

pérdida del primer molar. Para su diseño en el software de simulación Autodesk Inventor Professional 3D se obtuvo las medidas de dientes y hueso mandibular reales. De la misma manera, se diseñó los resortes cantilever de TMA, los brackets, tubos y arco de anclaje de acero inoxidable.

Fueron seleccionados 16 dientes reales de la arcada mandibular de un conjunto de dientes obtenido en el Centro de Salud Pública de Nayón. Con un calibrador digital marca TRUPER se procedió a medir las dimensiones de cada diente: alto (desde el borde incisal al ápice), ancho (de mesial a distal) y espesor (de vestibular a lingual); con el fin de realizar un cubo con dichas medidas en el software Autodesk Inventor Professional 3D.
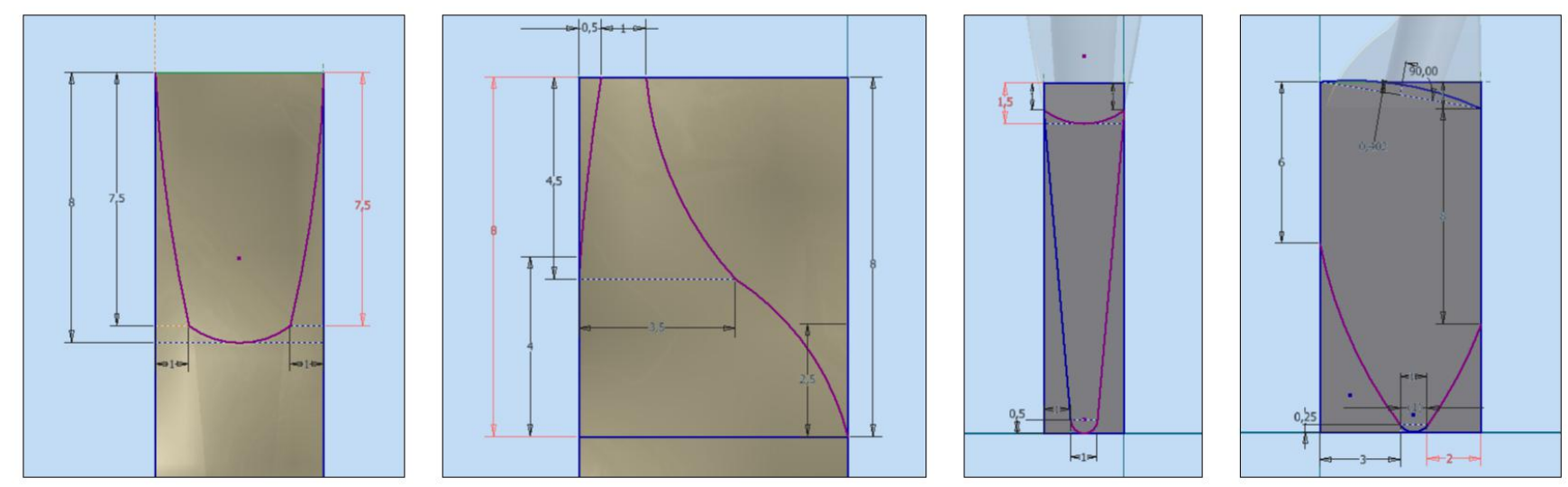

Figura 2. Diseño de caras vestibular y proximal corona y raíz de incisivo central inferior en el Software Autodesk Inventor Professional 3D. 


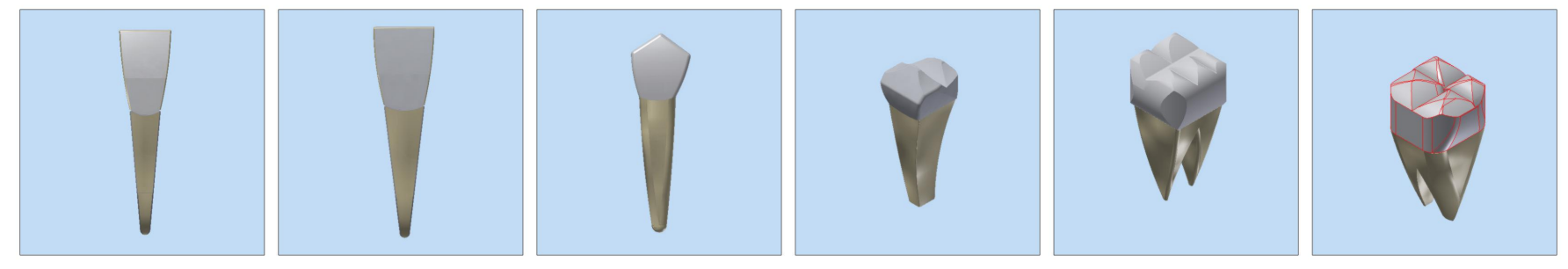

Figura 3. Diseño 3D de los dientes.

A partir de este cubo se empezó a diseñar mediante líneas rectas y curvas las diversas partes del diente dentro del cubo; primero de la cara vestibular, después de las áreas proximales y, por último, de la zona palatina. Cabe recalcar, que los dientes tienen diversas medidas y espesores de acuerdo a la zona; de manera que, para que la simulación sea fidedigna se tomaron minuciosamente las medidas de cada parte del diente.

Al final, mediante un comando del software se eliminaron las partes del cubo restantes que estaban fuera de las líneas trazadas y se obtuvo el diseño 3D de cada uno de los dientes de la arcada mandibular, desde incisivos hasta segundos molares exceptuando el primer molar No. 46.

Después de tener el diseño 3D de los dientes se diseñó la cavidad pulpar, los conductos y la pulpa de todos los dientes.

Asimismo, se obtuvo un hueso mandibular real del laboratorio de Anatomía de la Facultad de Odontología de la Universidad San Francisco de Quito. Para el diseño 3D del hueso, al igual que con los dientes, se midió minuciosamente con calibrador cada una de las estructuras y partes del mismo desde la apófisis coronoides en la parte posterior hasta el mentón en la parte anterior. Es así que, se formó el hueso mandibular en el software Autodesk Inventor Professional 3D mediante líneas rectas y curvas que abarcaron zonas como: la apófisis coronoides y parte de la rama, el cuerpo mandibular, el hueso alveolar, sínfisis y mentón. Рага obtener el modelo 3D del hueso, se utilizó un comando del software que permite convertir las diferentes caras dibujadas de la mandíbula en un objeto virtual. Tanto los dientes como la mandíbula se consideran dentro del programa como cuerpos rígidos. Para el diseño del alvéolo se realizó agujeros en el hueso alveolar virtual. Рara este efecto, se midió el contorno de la zona cervical y apical de los dientes virtuales y se dibujó dos círculos alrededor de la raíz del diente 3D separado del borde de la raíz virtual $0.20 \mathrm{~mm}$. 5,6 De esta manera, alrededor de la raíz se tiene dos círculos uno más grande en la parte cervical de la raíz virtual y uno pequeño en la parte apical. Dichos círculos se unen entre sí mediante un comando del software, una vez unidos se utiliza otra función del programa para que entre estos dos círculos se forme un hueco dentro del hueso. La separación de $0.20 \mathrm{~mm}$ se la realizó con el fin de que el espacio entre la raíz virtual y el hueso alveolar sea ocupado por el ligamento periodontal virtual. En el año 2005, según Yukio Kojima para la simulación del ligamento periodontal se asume que este es una membrana elástica y homogénea con espesor uniforme, de manera que al aplicar la fuerza sobre los dientes virtuales éste se deforme un poco y actúe como un resorte elástico. ${ }^{6}$ En este estudio se lo diseñó de la misma manera para todos los dientes. Una vez realizadas las estructuras anatómicas, en el mismo software se diseñaron mediante líneas rectas y curvas los brackets y tubos tanto simples como dobles de slot $0.022^{\prime \prime} \times 0.028^{\prime \prime}$ con $0^{\circ}$ de tip y torque. Al igual que los dientes y el hueso, se obtuvieron brackets y tubos edgewise reales y se midieron sus diversas dimensiones de la base y de 


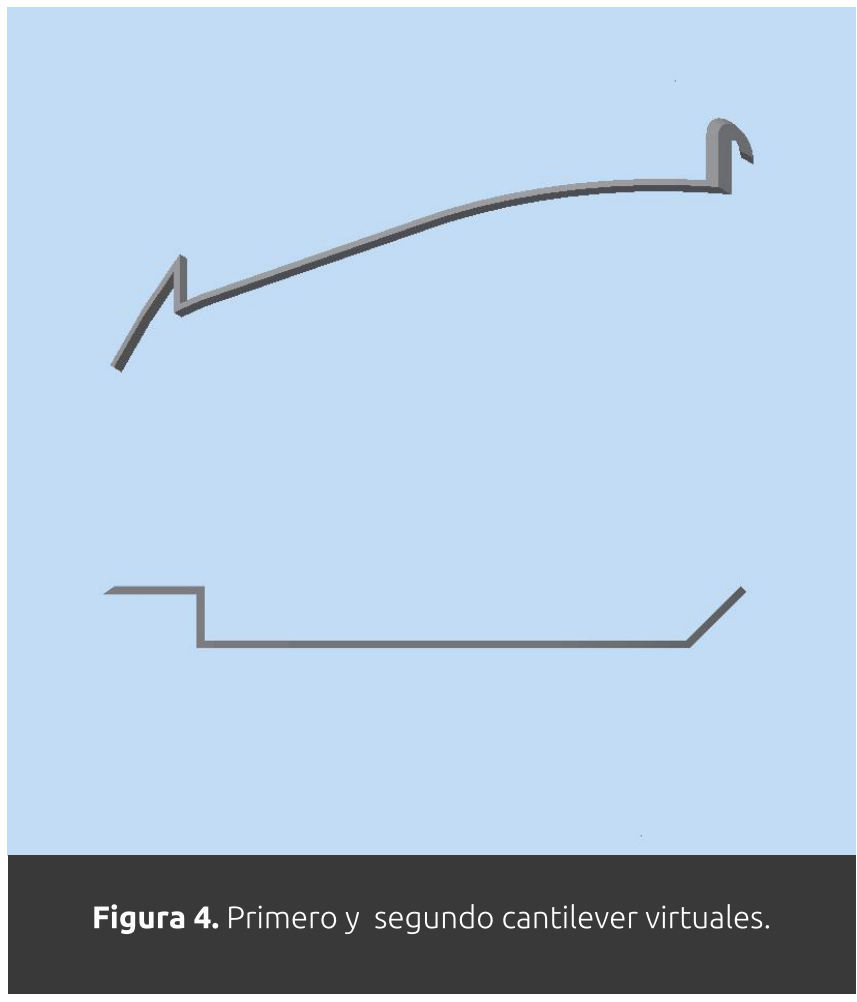

la ranura: alto, ancho, espesor con un calibrador. Además, se diseñaron también dos cantilever de sección 0.017"x 0.025". El primer cantilever tiene una activación tip back de $45^{\circ}$ y una longitud de $30 \mathrm{~mm}$; mientras que el segundo cantilever, cuyo objetivo es evitar la extrusión del molar durante la verticalización, se lo diseñó con una activación de $45^{\circ}$ en el brazo anterior. ${ }^{7}$ Ambos cantilever se realizaron con la misma curvatura del arco de alambre $0.019 \times 0.025^{\prime \prime}$ utilizado como anclaje. Por otro lado, también se dibujó un tubo criss-cross con sus dimensiones exactas, del cual emerge el segundo cantilever. Por último, se dibujó un arco de acero inoxidable de sección $0.019^{\prime \prime} \times 0.025^{\prime \prime}$ con las mismas medidas de un arco $0.019 \times 0.025$ real. Este se usará como anclaje. Todas estas estructuras virtuales diseñadas por separado se deben unir mediante un comando del software. Los dientes fueron colocados dentro de sus alveolos con la separación de $0.20 \mathrm{~mm}$ correspondiente al espacio del ligamento periodontal. 5,6 Asimismo, los brackets fueron colocados en la mitad de la corona clínica de premolar a premolar, en el segundo molar (No. 47) mesioinclinado se situó el tubo doble y en los molares del lado contrario (No. 36 y 37) los tubos

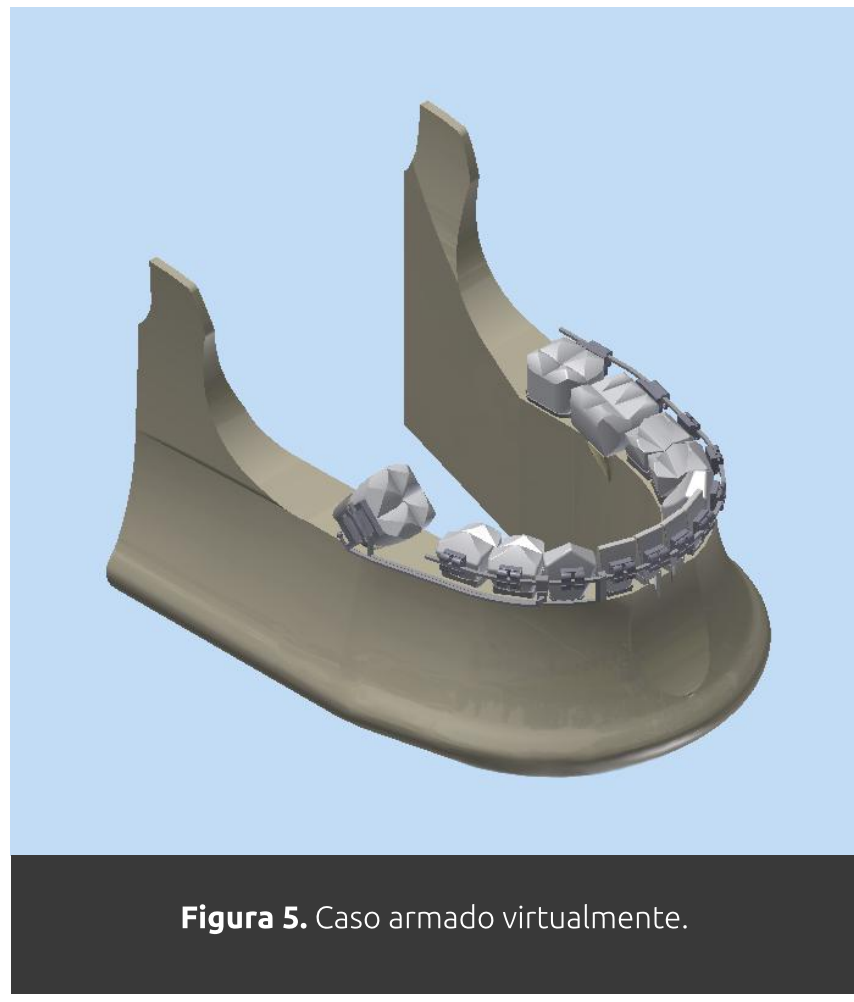

simples. El arco de sección $0.019 \times 0.025^{\prime \prime}$ se colocó en el slot de los tubos y brackets desde el molar 37 al premolar 45. El tubo criss-cross se localizó entre el canino 43 y el premolar 44. Por último, el primer cantilever parte desde el tubo del molar 47 y se engancha en el arco $0.019 x$ 0.025" entre canino (No. 43) y lateral (No. 42). El segundo cantilever sale desde el tubo criss-cross para sujetarse en el tubo doble del molar 47.

Para que la simulación se asemeje a la realidad el software Autodesk Inventor Professional requiere de información; por ejemplo, propiedades físicas como: el módulo de elasticidad y el coeficiente de Poisson de todos

\begin{tabular}{|l|l|l|}
\hline \multicolumn{3}{|l|}{ PROPIEDADES MECÁNICAS DE LOS MATERIALES USADOS EN EL ESTUDIO } \\
\hline MATERIAL & MÓDULO DE YOUNG & COEFICIENTE DE POISSON \\
\hline Esmalte & $20 \mathrm{GPa}$ & 0.3 \\
\hline Cemento & $18.6 \mathrm{MPa}$ & 0.3 \\
\hline Dentina & $18.6 \mathrm{MPa}$ & 0.3 \\
\hline Pulpa & $2.7 \mathrm{MPa}$ & 0.4 \\
\hline Ligamento periodontal & $0.71 \mathrm{MPa}$ & 0.4 \\
\hline Hueso & $345 \mathrm{MPa}$ & 0.3 \\
\hline Acero inoxidable & $200 \mathrm{GPa}$ & 0.3 \\
\hline TMA & $69 \mathrm{GPa}$ & 0.3 \\
\hline
\end{tabular}

Tabla 1. Propiedades mecánicas de los materiales usados en el estudio. Obtenido de (Caballero, y otros, 2015) (Рагга \& Rivadeneira, 2013) 


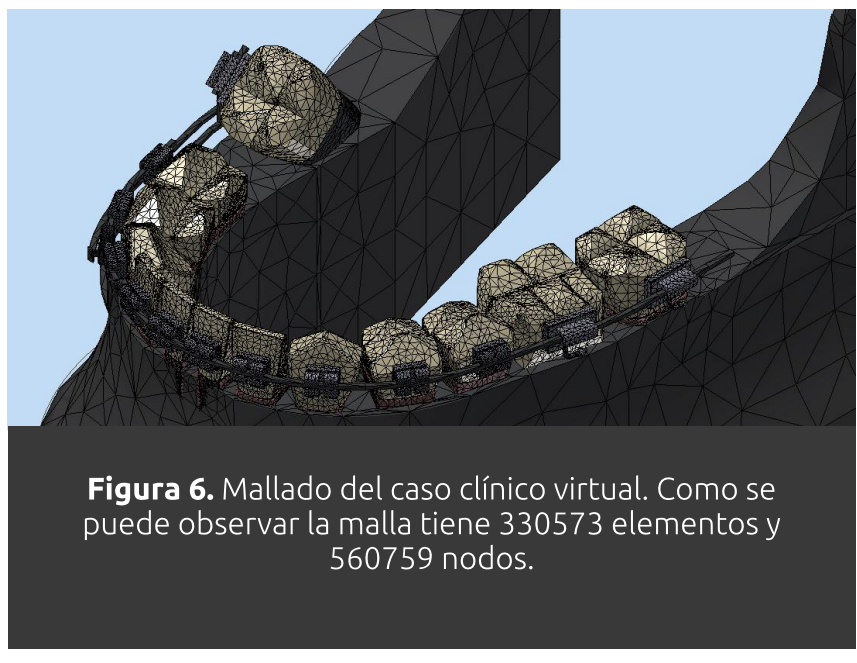

los materiales simulados; lo cual se basó en estudios anteriores:

El alambre de sección $0.019 \times 0.025^{\prime \prime}$, los brackets, tubos dobles, tubos simples y tubo criss-cross mantienen las propiedades físicas del acero inoxidable. Los cantilever de sección 0.017 x 0.025" se regirán por las propiedades del TMA. $Y$ los dientes, hueso y ligamento periodontal tendrán sus respectivas características según la tabla anterior. Una vez ingresados los datos de propiedades mecánicas de los materiales y asignados a sus respectivos objetos virtuales, se procede a realizar el mallado del caso clínico virtual mediante el comando Mesh View. Como se mencionó anteriormente este paso consiste en crear la malla contenida por elementos y nodos sobre el objeto diseñado en el programa. Dichos elementos van a ser aquellos que reciban la información de esfuerzos, tensiones y compresiones cuando se realice la simulación.

De acuerdo al autor Gonzalo Uribe en el año 2010, el momento ideal de verticalización es de $1.200 \mathrm{gf} \cdot \mathrm{mm}$; de manera que para que esto se cumpla se utilizó una fuerza de $40 \mathrm{gf}$ con una longitud del primer cantilever de $30 \mathrm{~mm}$. Asimismo, el segundo cantilever, al ser más corto, realiza una fuerza un tanto mayor a la del primero. Рara conocer la cantidad de fuerza que realiza el segundo cantilever se dobló un alambre TMA real con las mismas características del cantilever virtual y se midió la fuerza con un dinamómetro, lo cual dio como resultado 43gf. Las interacciones entre los brackets y el alambre fueron determinadas usando elementos beam. Las interacciones remanentes entre los elementos de diferentes objetos fueron hechas mediante interacciones de contacto rígido, en el que las fases de los diferentes materiales permanecen sin desplazamiento relativo entre ellos; es decir, no se pueden separar. Estas interacciones de contacto rígido se mantienen entre las coronas de los dientes, los brackets y tubos para semejar la adhesión entre estos elementos en un caso real; igualmente, se mantuvo la interacción entre los brackets, tubos y los alambres con el fin de simular el ligado metálico o elástico. Se usó tres ejes de referencia para determinar los movimientos del molar: 1) eje x рага movimientos bucolinguales, 2) eje y рага movimientos oclusogingivales y 3) eje z para movimientos mesiodistales. Además, se realizó también un análisis del estrés que sufren las diferentes partes del diente cuando se aplica el sistema de fuerzas.

Se va a realizar un total de 4 simulaciones con diferentes tipos de mallas; es decir, cada malla va a tener una mayor cantidad de elementos de manera que va a obtener datos más precisos. Los datos que se obtengan de las mismas sobre movimientos en los diferentes ejes ya mencionados y los datos sobre esfuerzos en las diferentes partes del diente serán analizados mediante estadística descriptiva y de centralización.

\section{Resultados}

Análisis de movimientos en los ejes $X, Y, Z$ : Se realizó 4 simulaciones con diferentes tipos de mallas. La malla contiene elementos y nodos que calculan la información requerida sobre movimientos y esfuerzos mediante ciertas operaciones matemáticas. Cada malla contiene una mayor cantidad de elementos y nodos, por lo que la simulación realiza más operaciones y los datos que se obtienen son más precisos. Las 


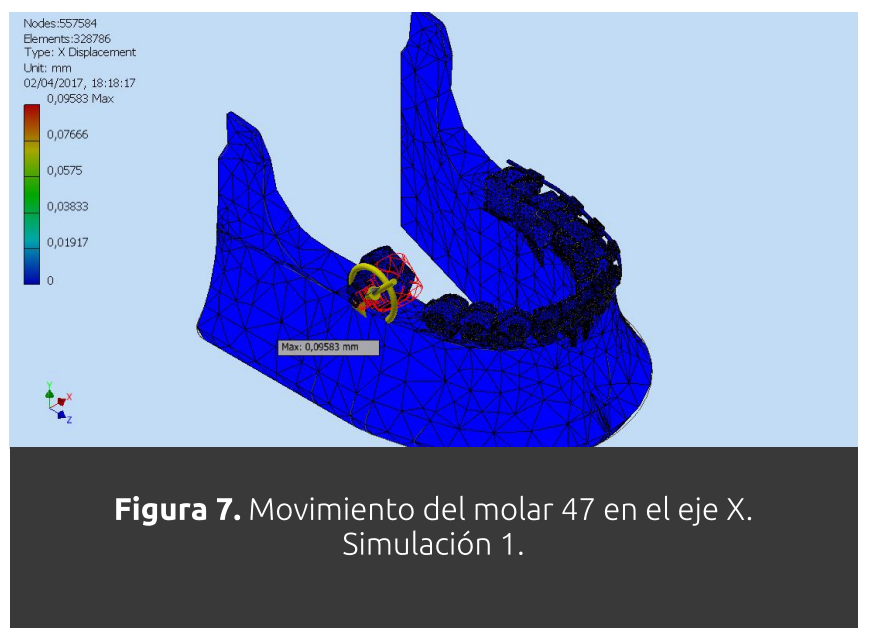

simulaciones muestran el primer movimiento del molar por lo que en algunas imágenes no se va a observar el molar verticalizado completamente.

\section{Simulación 1}

Eje X: El eje $X$ determina movimientos en sentido bucolingual; es decir, corrobora movimientos hacia vestibular o lingual del molar a verticalizar después de aplicar el sistema de fuerzas. La posición original del molar se muestra por las líneas dibujadas de color rojo, mientras que la nueva posición de la pieza es la imagen que tiene color. Los diferentes colores de la barra a la izquierda de las imágenes informan sobre la cantidad de movimiento que tiene cada parte del molar. Por ejemplo, el color rojo significa la mayor cantidad de movimiento o movimiento máximo, lo cual corresponde al valor de $0.09583 \mathrm{~mm}$ perteneciente a la parte oclusal de la corona. (Figura 7)
Eje Y: El eje $\mathrm{Y}$ muestra los movimientos oclusogingivales del molar 47. Aquí se puede observar un movimiento de intrusión del molar de $0.1832 \mathrm{~mm}$ como movimiento máximo que está representado en el ápice de la raíz distal. La zona oclusomesial de la corona representa un movimiento de $0.0366 \mathrm{~mm}$. (Figura 8)

Eje Z: El eje Z indica el movimiento mesiodistal de la corona. El movimiento máximo del molar en sentido mesiodistal fue de $0.142 \mathrm{~mm}$. El color rojo que se puede observar en la parte distooclusal de la corona representa dicho movimiento máximo. El color azul ubicado en la parte mesial de la corona indica un movimiento de $0 \mathrm{~mm}$. (Figura 9)

\section{Simulación 2.}

Eje X: Este eje demuestra que existe un ligero movimiento del molar 47 hacia vestibular; 0.4786mm exactamente. (Figura 10)

Eje Y: En el eje Y se puede evidenciar que la pieza se intruye $0.4375 \mathrm{~mm}$ lo cual representa el máximo movimiento. En la imagen se puede ver que la parte distal de corona y raíz se intruye mayormente. El movimiento mínimo se encuentra en la parte media a mesial del diente completo. (Figura 11)

Eje Z: Por otro lado, en el eje Z, la parte oclusal de la corona sufre un mayor movimiento hacia distal; $1.476 \mathrm{~mm}$. La parte media del diente se mueve $0.59 \mathrm{~mm}$ hacia el mismo lado; mientras
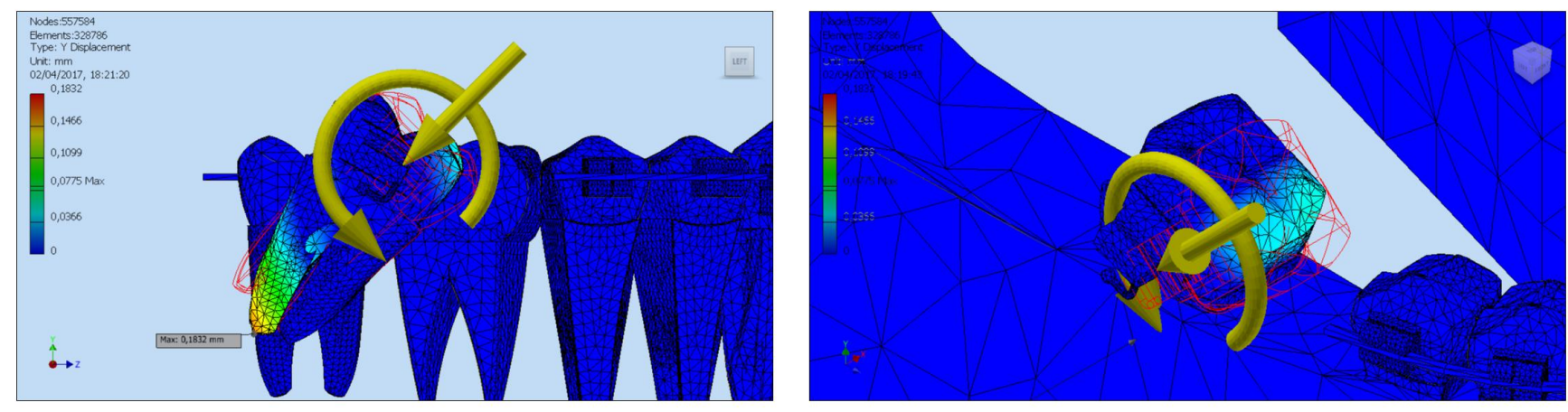

Figura 8. Movimiento del molar 47 en el eje Y. Simulación 1. Simulación 1. 

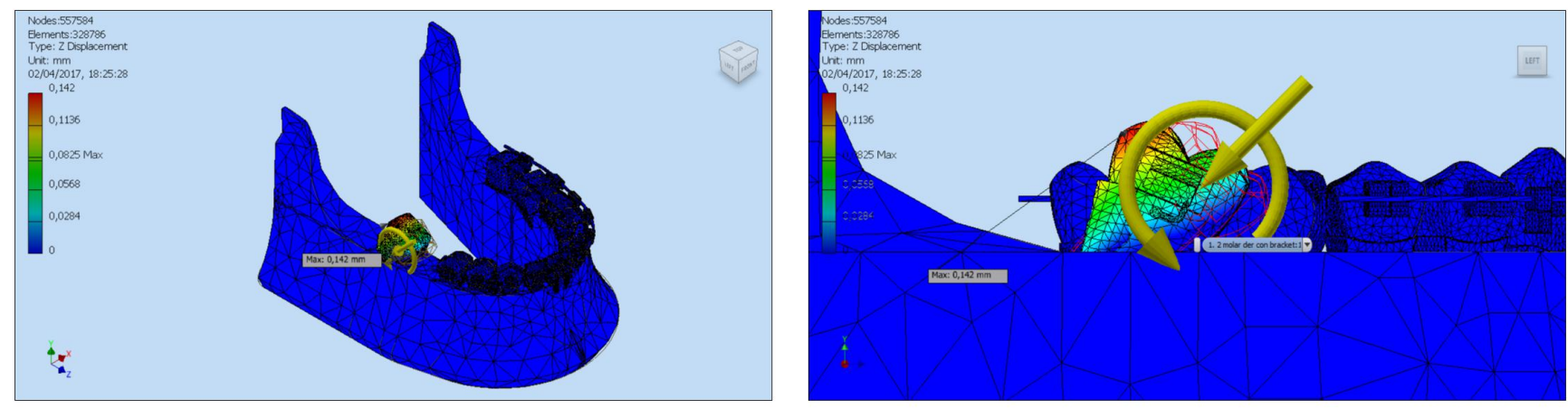

Figura 9. Movimiento del molar 47 en el eje Z. Simulación 1.

que la parte media y apical de la raíz indica un movimiento de $0.022 \mathrm{~mm}$. (Figura 12)

\section{Simulación 3.}

Eje X: En la tercera simulación se puede observar un movimiento del molar 47 en el eje $X$ de $0.5121 \mathrm{~mm}$ hacia vestibular.

Eje Y: El eje Y muestra el movimiento de intrusión del molar, que según esta simulación es de $1.308 \mathrm{~mm}$. Cabe recalcar que la zona apical de la raíz distal es aquella que sufre mayor movimiento. El menor movimiento de intrusión se da en el área oclusomesial de la corona, lo cual corresponde a $0.262 \mathrm{~mm}$.

Eje Z: En este eje el movimiento del molar es de $1.44 \mathrm{~mm}$ hacia distal, lo cual muestra que el cantilever está cumpliendo su función de verticalización. El menor movimiento lo sufre la zona medial y apical de la raíz.

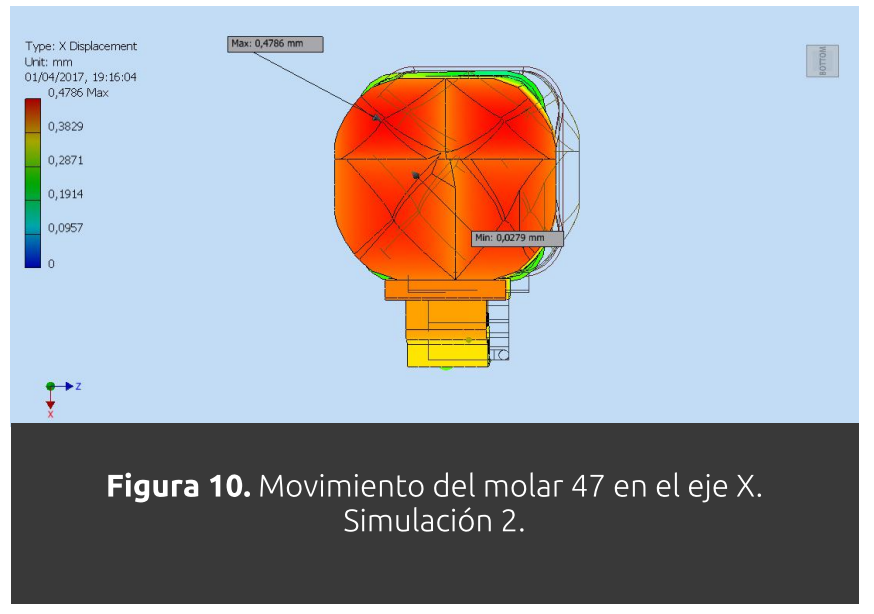

\section{Simulación 4.}

Eje X: La simulación 4 muestra un mayor movimiento del molar en el eje $X$ con respecto a las demás simulaciones. La corona presenta el movimiento máximo hacia vestibular que es de $1.153 \mathrm{~mm}$ hacia vestibular. La raíz, en este caso, presenta un movimiento de $0.023 \mathrm{~mm}$. Si se compara con las demás simulaciones la raíz no presentaba movimiento en el eje $\mathrm{X}$.

Eje Y: El movimiento de intrusión en el eje Y también es mayor en esta simulación; sin embargo, el desplazamiento máximo de $3.253 \mathrm{~mm}$ está solamente en la zona oclusomesial de la corona, mientras que en las simulaciones anteriores el movimiento mayor se encontraba en el ápice de la raíz distal también. En este caso el ápice de la raíz distal presenta un movimiento de $0.651 \mathrm{~mm}$.

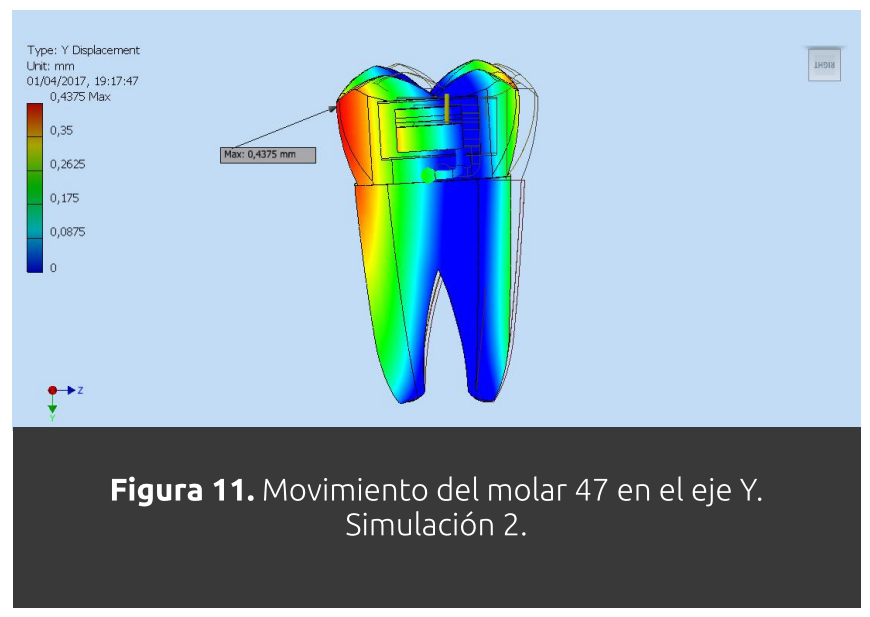




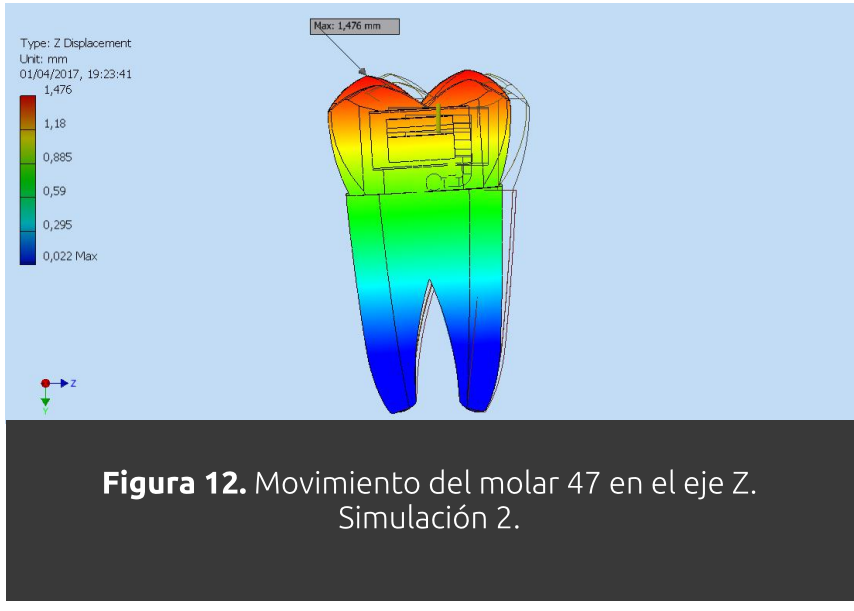

Eje Z: El movimiento en el eje $Z$ muestra un desplazamiento distal de la corona de $4.139 \mathrm{~mm}$ hacia distal.

Con los datos de las simulaciones se realizó un análisis estadístico descriptivo, el cual se lo puede visualizar en la tabla 2.

Análisis de esfuerzos: Рага el análisis de esfuerzos el programa realizó operaciones matemáticas similares a las de movimiento, como aquellas usadas en la Tensión de Von Mises o Von Mises Stress, para obtener la cantidad de fuerza que recibe ciertas zonas del diente. En este estudio se realizaron varias simulaciones en donde se adquirieron imágenes de las diferentes partes del molar que al aplicar el sistema de fuerzas sufrieron esfuerzos de tensión y compresión. Asimismo, las mallas se cambiaron con cada simulación para obtener datos más precisos.

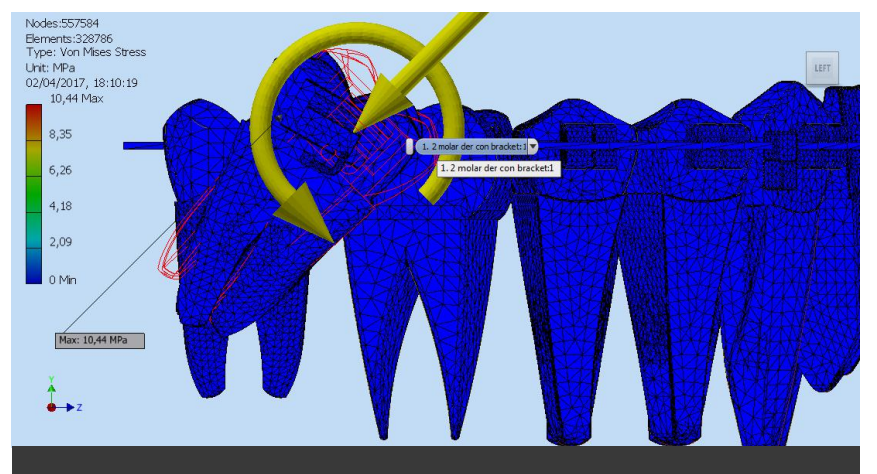

Figura 13. Análisis de esfuerzos. Simulación 1.

\begin{tabular}{|l|l|l|l|}
\hline \multicolumn{4}{|c|}{ TABLA DE SIMULACIÓN DE MOVIMIENTO DEL MOLAR 47 EN LOS EJES X, Y, Z } \\
\hline & Eje X & Eje Y & Eje Z \\
\hline Simulación 1 & $0.09583 \mathrm{~mm}$ & $0.1832 \mathrm{~mm}$ & $0.142 \mathrm{~mm}$ \\
\hline Simulación 2 & $0.4786 \mathrm{~mm}$ & $0.4375 \mathrm{~mm}$ & $1.476 \mathrm{~mm}$ \\
\hline Simulación 3 & $0.5121 \mathrm{~mm}$ & $1.308 \mathrm{~mm}$ & $1.44 \mathrm{~mm}$ \\
\hline Simulación 4 & $1.153 \mathrm{~mm}$ & $3.253 \mathrm{~mm}$ & $4.139 \mathrm{~mm}$ \\
\hline Media de movimiento & $0.5598 \mathrm{~mm}$ & $1.2954 \mathrm{~mm}$ & $1.7992 \mathrm{~mm}$ \\
\hline Mediana & $0.4953 \mathrm{~mm}$ & $0.8727 \mathrm{~mm}$ & $1.458 \mathrm{~mm}$ \\
\hline
\end{tabular}

Tabla 2. Cantidad de movimiento en los ejes $X, Y, Z$

\section{Simulación 1.}

En la simulación 1 se obtuvo un esfuerzo promedio de 10.44MPa o $1.06 \mathrm{Kg} / \mathrm{mm}^{2}$ al aplicar el sistema de fuerzas. El estrés se ubica específicamente en la parte distal del bracket como muestra la flecha en la imagen. También se obtuvo un esfuerzo en la parte apical de la raíz distal de aproximadamente 0.01MPa o 0.00102 $\mathrm{Kg} / \mathrm{mm}^{2}$. (Figura 13). Además, se analizó un factor de seguridad. Este factor representa los límites de presión que puede soportar un material antes de sufrir un efecto indeseable, como rotura, basado en la fuerza aplicada. En el caso del diente podría producirse una descementación del bracket o reabsorción de la raíz. En la figura la barra de colores representa la imagen entre el límite mínimo con 0.14 y el límite máximo de 15 (el factor no presenta unidades). Mientras menor sea el factor de seguridad existe más probabilidad de que exista un efecto indeseable. El límite mínimo representado por el color rojo se encuentra en los alrededores del bracket, lo cual quiere decir que con la fuerza empleada en el sistema puede haber la

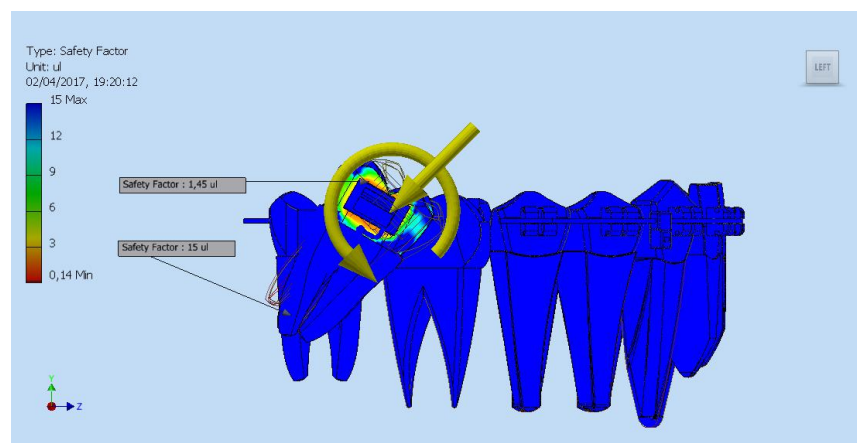

Figura 14. Factor de Seguridad. 

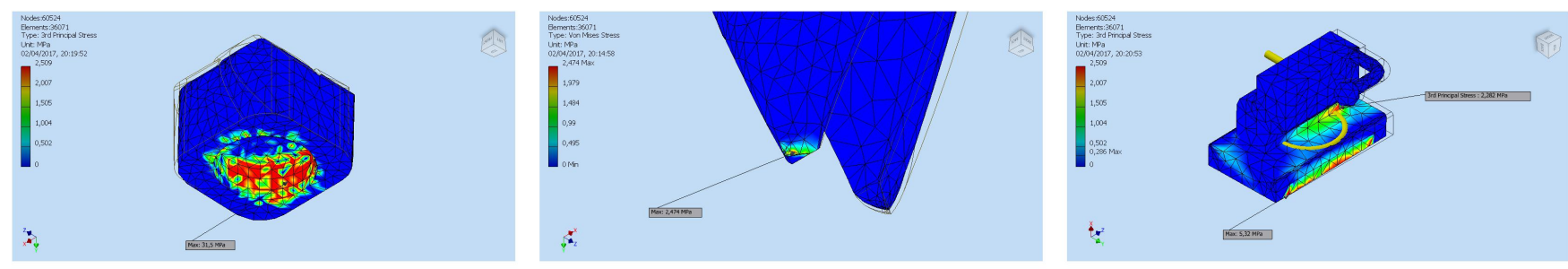

Figura 15. Estrés en compresión en la corona, raíz y tubo. Simulación 2.

posibilidad del que el tubo se descemente. Por otro lado, el límite máximo significa que hay la posibilidad de aumentar la fuerza pues no se va a dar un efecto indeseable. Este límite es de color azul y según la figura, la raíz no sufre ningún daño con la fuerza y el momento aplicados. (Figura 14)

\section{Simulación 2.}

En la simulación de esfuerzos número 2 se realizó un análisis detallado del estrés en tensión y en compresión que sufren las diferentes partes del diente con la fuerza aplicada en el sistema.

Estrés en Tensión: El estrés en tensión significa la cantidad de esfuerzo que sufre el material con fuerzas de tensión. En la corona, específicamente en la cavidad pulpar existe un esfuerzo de tensión en un punto de la pared mesial de aproximadamente $48.77 \mathrm{MPa}$ o $4.97 \mathrm{Kg} / \mathrm{mm}^{2}$. En cuanto a la raíz, se obtuvo un esfuerzo máximo en el ápice de 2,474MPa o $0.25 \mathrm{KgF} / \mathrm{mm}^{2}$. El tubo también fue objetos de análisis, de manera que en la simulación 2 se muestra un esfuerzo máximo de 5.134MPa o $0.52 \mathrm{kgF} / \mathrm{mm}^{2}$ en la zona baja de la base, mientras que en los extremos superiores del tubo se encontró un estrés de 2.069MPa o $0.211 \mathrm{kgf} / \mathrm{mm}^{2}$.
Estrés en Compresión: El estrés en compresión significa el esfuerzo que sufre el material con fuerzas de compresión.

La cavidad pulpar sufre un gran esfuerzo con fuerzas de compresión. La presión máxima es de $31.5 \mathrm{MPa}$ o $3.213 \mathrm{~kg} / \mathrm{mm}^{2}$. La fuerza de compresión también está presente en el ápice de la raíz distal, lo cual es esperado ya que ésta sufre una intrusión. El esfuerzo máximo que sufre el ápice es de 2.509MPa o $0.255 \mathrm{Kgf} / \mathrm{mm}^{2}$. La raíz mesial sufre poco esfuerzo con 0,088MPa o 0.008 $\mathrm{Kgf} / \mathrm{mm}^{2}$. Por último, en el tubo existen dos áreas que sufren esfuerzo en compresión. La primera está en la unión del tubo con la base que sufre un estrés de 2.282MPa o $0.23 \mathrm{kgF} / \mathrm{mm}^{2}$, mientras que el segundo está en la base y siente $5.32 \mathrm{MPa}$ o $0.54 \mathrm{Kg} f / \mathrm{mm}^{2}$ de presión. (Figura 15)

\section{Simulación 3.}

En la simulación 3 se realizó nuevamente un análisis de los esfuerzos en tensión y en compresión que sufren las diferentes partes del diente.

Estrés en tensión: La simulación 3 muestra un esfuerzo máximo en tensión de la parte interna
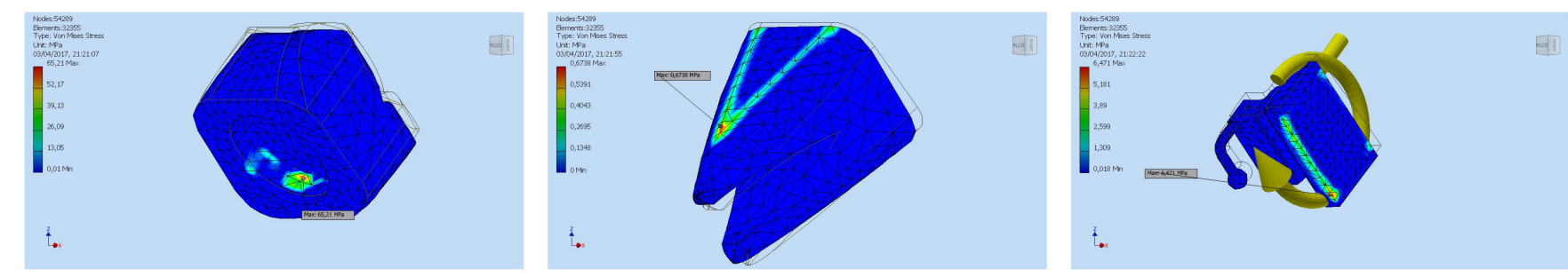

Figura 16. Estrés en tensión de la corona, raíz y tubo. Simulación 3. 

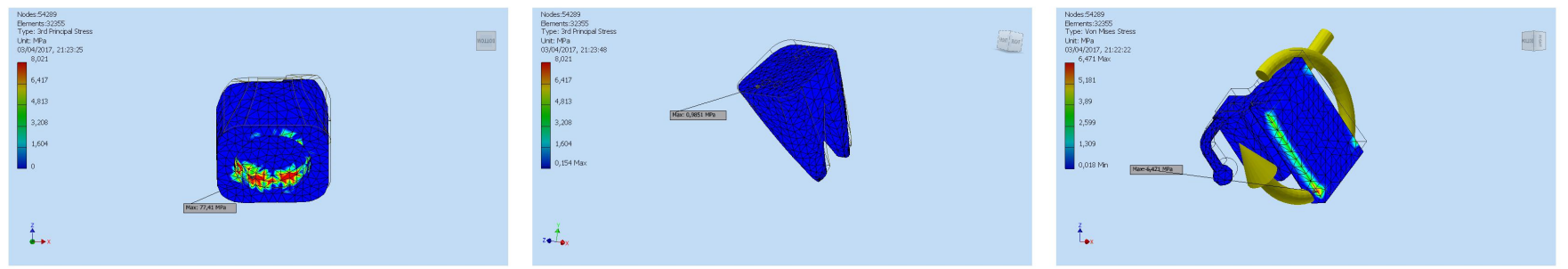

Figura 17. Estrés en tensión de corona, raíz y tubo. Simulación 3.

del diente, específicamente en la cavidad pulpar. El valor de tensión que esta área siente es de 65.21 MPa o $6.65 \mathrm{kgf} / \mathrm{mm}^{2}$. En este caso, la raíz sufre un esfuerzo máximo de $0.6738 \mathrm{MPa}$ o 0.068 $\mathrm{Kgf} / \mathrm{mm}^{2}$. El tubo, por su parte, solamente sufre un punto de tensión en el extremo de la parte distal de la base correspondiente a 6.471 MPa o $0.66 \mathrm{Kgf} / \mathrm{mm}^{2}$. Asimismo, en el borde inferior de la base existe $2.599 \mathrm{MPa} 00.265 \mathrm{Kg} / \mathrm{mm}^{2}$ de tensión. (Figura 16)

Estrés en compresión: La simulación 3 corrobora lo que se observó en la simulación 2, ya que muestra que la cavidad pulpar sufre grandes presiones. En este caso la compresión máxima fue de 77.41MPa o $7.895 \mathrm{Kgf} / \mathrm{mm}^{2}$. Esta evidencia puede significar que la pulpa tiene la posibilidad de verse afectada con la fuerza aplicada en este sistema cantilever doble. Esta vez en la raíz se muestra un 0.9851MPa o 0.1004 $\mathrm{Kgf} / \mathrm{mm}^{2}$ de presión en la zona cervical de la raíz; mientras que en la simulación anterior la presión se encontraba en el ápice. Adicionalmente, el tubo sufre una presión de $8.021 \mathrm{MPa}$ o 0.818 $\mathrm{Kgf} / \mathrm{mm}^{2}$ en el mismo sitio que mostró la simulación 2. (Figura 17)

\section{Discusión}

La verticalización de molares es un tratamiento que se requiere en dos situaciones específicas: cuando se pierde un molar y el diente adyacente se mesioinclina hacia el espacio y por impactación de molares permanentes. Ambas condiciones representan un desafío para el ortodoncista cuyo objetivo es verticalizar el molar para mejorar la salud oral y facial del paciente. ${ }^{\mathbf{8}, \mathbf{9}}$

Esta investigación se centró en el estudio de verticalización de molares cuando existe pérdida del primer molar y, en consecuencia, el segundo molar se mesioinclina hacia el espacio. Рara el enderezamiento de estos molares se han

\begin{tabular}{|l|l|l|l|l|}
\hline TABLA DE DATOS DE ESTRÉS EN EL SEGUNDO MOLAR (KgF/mm2) & \multicolumn{3}{l|}{ Simulación 2 } & \multicolumn{1}{l|}{ Simulación 3} \\
\hline $\begin{array}{l}\text { Tensión Von Mises } \\
\text { (Promedio) }\end{array}$ & $\begin{array}{l}\text { Simulación 1 } \\
1.06 \mathrm{Kgf} / \mathrm{mm}^{2} \text { en } \\
\text { distal del bracket } \\
0.00102 \mathrm{Kgf} / \mathrm{mm}^{2} \text { en } \\
\text { el ápice }\end{array}$ & & \\
\hline Estrés en Tensión & Corona & & $4.97 \mathrm{Kgf} / \mathrm{mm}^{2}$ & $6.65 \mathrm{Kgf} / \mathrm{mm}^{2}$ \\
\hline & Raíz & & $0.25 \mathrm{Kgf} / \mathrm{mm}^{2}$ & $0.068 \mathrm{Kgf} / \mathrm{mm}^{2}$ \\
\hline & Tubo & & $0.52 \mathrm{Kgf} / \mathrm{mm}^{2}$ & $0.66 \mathrm{Kgf} / \mathrm{mm}^{2}$ \\
\hline $\begin{array}{l}\text { Estrés en } \\
\text { compresión }\end{array}$ & Corona & & $3.213 \mathrm{Kgf} / \mathrm{mm}^{2}$ & $7.895 \mathrm{Kgf} / \mathrm{mm}^{2}$ \\
\hline & Raíz & & $0.255 \mathrm{Kgf} / \mathrm{mm}^{2}$ & $0.1 \mathrm{Kgf} / \mathrm{mm}^{2}$ \\
\hline & Tubo & $0.23 \mathrm{Kgf} / \mathrm{mm}^{2}$ & $0.818 \mathrm{Kgf} / \mathrm{mm}^{2}$ \\
\hline
\end{tabular}


utilizado varios sistemas de fuerzas que en su mayoría son extrusivos, lo cual se considera un efecto adverso sobretodo en pacientes dolicofaciales, como se lo menciona en varios estudios. $\mathbf{8 , 1 0 , 1 1 , 1 2 , 1 3}$ Los sistemas físicos estáticamente determinados son aquellos que proporcionan un control preciso de fuerzas y momentos. Los cantilevers, sugeridos por Weilan y Batleon en 1992, son los sistemas estáticamente determinados más representativos y se encuentran dentro del grupo de aparatos de verticalización con componente extrusivo. El cantilever se usa entre dos puntos alejados entre sí con un extremo fijo insertado en un tubo y el otro libre que se apoya en la unidad de anclaje con un punto de contacto. El momento ideal que debe realizar el cantilever es de $1.200 \mathrm{gf} . \mathrm{mm}$. "Si la fuerza de intrusión que produce un resorte de verticalización es de $40 \mathrm{gf}$ sobre el arco base y la longitud es de $30 \mathrm{~mm}$, el momento de desinclinación en el molar será de 1.200gf.mm." 7

Dicho sistema de fuerzas fue tomado en cuenta para este estudio; sin embargo, el resorte utilizado en la simulación fue el cantilever doble, que consiste en el uso de dos cantilevers: el uno para verticalizar y el segundo para impedir la extrusión del molar. Con el uso de estos dos cantilevers y el sistema de fuerzas ya mencionado se obtuvo un resultado favorable en las simulaciones realizadas en este estudio ya que no solamente se evitó la intrusión del molar 47 sino que también hubo un movimiento de intrusión del mismo de $1.29 \mathrm{~mm}$. La presente investigación coincide con el estudio de Locks, Locks y Locks, el cual describe, en el año 2015, que uno de los métodos para impedir la extrusión del molar es la combinación de dos cantilevers, de los cuales el uno se engancha en el segmento anterior entre canino e incisivo lateral y el segundo sale de un tubo criss-cross que se coloca entre canino y premolar o entre premolares y se engancha en el segmento posterior al molar inclinado; de esta manera, el segundo cantiléver contrarresta la fuerza extrusiva del primero en el molar donde se aplica la fuerza. Sakima en 1999 también describe al cantilever doble propuesto por Weiland \& col. y Melsen \& col. como un sistema de fuerzas útil para la verticalización del molar con la ventaja de promover la intrusión del mismo. La revisión bibliográfica realizada por Cascaes en el año 2014 cita a los autores Shellhart y Oesterle que en el año 1999 concluyen que el uso de cantilever doble es la técnica más difundida usada para verticalización sin extrusión. A más de ello en el mismo año, Cascaes también menciona que, según Melo en 2011, el sistema cantilever doble está indicado para una verticalización con intrusión del molar. ${ }^{10,11,12}$

Lamentablemente no existen mayor cantidad de estudios que describan la biomecánica del cantilever doble y que corroboren la cantidad de intrusión que se puede lograr con este sistema; es por ello que esta investigación se enfocó en descubrir cuánto movimiento y que efectos produce el cantilever doble sobre el molar.

Por otro lado, este estudio también obtuvo un análisis del estrés que sufre cada parte del diente con fuerzas de tensión y compresión al aplicar el sistema antes mencionado. Lo que llamó la atención de este análisis es que varias paredes de la cavidad pulpar reciben mayor cantidad de estrés comparado con otras partes del diente. Dicha presión se dio tanto en tensión como en compresión al aplicar el resorte cantilever doble. Por otro lado, el ápice de la raíz no mostró un estrés considerable con el uso de estas fuerzas a pesar de la intrusión que se logró obtener. Cabe recalcar que esta parte del estudio es inédita ya que no existen investigaciones realizadas de este tipo que hayan usado el método de elementos finitos.

Una de las limitaciones de este estudio fue la selección de los dientes por cuanto en el centro de salud tenían un frasco con dientes de varios pacientes sin distinción de edad o género; sin embargo, de entre el conjunto de dientes se seleccionó piezas con características similares de 
desgaste y coloración, así como también con medidas de alto, ancho y espesor comunes en la mayoría de pacientes.

\section{Conclusiones}

El sistema cantilever doble evita la extrusión del molar durante el movimiento de verticalización del mismo.

El sistema cantilever doble produce un movimiento de intrusión promedio de $1.29 \mathrm{~mm}$ al realizar la verticalización de un molar.

Según el análisis del "Safety Factor", la fuerza de 40gf y el momento de 1200gf.mm aplicados en el sistema cantilever doble simulado no producen efectos adversos en la raíz de los molares e inclusive el estudio sugirió se puede aumentar la fuerza.

La fuerza aplicada al sistema produce estrés tanto en tensión como en compresión en diferentes partes del diente y del tubo, рего sobretodo en las paredes de la cavidad pulpar.

El estudio realizado ha demostrado que el sistema cantilever doble puede ser útil para verticalización de molares en pacientes en que se debe evitar la extrusión molar, ya que no solamente produce dicho efecto, sino que también provoca intrusión de la pieza.

\section{Referencias Bibliográficas}

1. Majourau, A., \& Norton, L. Uprighting impacted second molars with segmented springs. American Journal of Orthodontics and Dentofacial Orthopedics. 1995; 107: 235-238.

2. Souzal, I., Kumar, K., \& Shetty, S. Uprighting of molars-A clinical challenge. Virtual Journal of Orthodontics. 2013: 1-18.

3. Harfin, J. Tratamiento ortodóntico en el adulto. 2da Edición. Buenos Aires: Editorial Médica Panamericana; 2005.
4. Oliveira, A., Melo Pithon, M., \& Lacerda dos Santos, R. Miniscrew-supported coil spring for molar uprighting: Description. Dent Press J Orthod. 2013; 18(1): 45-49.

5. Caballero, G., Abadia de Carvalho, O., Oliveira, B., de Araujo Brito, H., Almeida, P., \& Oliveira, D. Mandibular canine intrusion with the segmented arch technique: A finite element method study. American Journal of Orthodontics and Dentofacial Orthopedics. 2015; 147: 691-697.

6. Kojima, Y., Mizuno, T., \& Fukui, H. A numerical simulation of tooth movement produced by molar uprighting spring. American Journal of Orthodontics and Dentofacial orthopedics. 2005;132: 631638.

7. Uribe, G. Ortodoncia: Teoría y Clínica. 2da Edición. Medellín: Corporación para investigaciones biológicas; 2010.

8. Valverde, R., \& Talavera, C. Verticalización de molares-Preparación ortodóncica del paciente protésico. Rev Estomatol Herediana. 2005; 15(2): 155-160.

9. Yáñez, E. E. Arte de la ortodoncia aplicada: Tomo 2. 1ra Edición. Venezuela: Amolca; 2015.

10. Locks, A., Locks, R. L., \& Locks, L. L. Diferentes abordagens para a verticalizacao de molares. Rev Clin Ortod Dental Press. 2015; 14(4): 32-48.

11. Sakima, T. Mechanical alternatives for molar uprighting. Force systems released by appliances. Revista Dental Press de Ortodoncia y Ortopedia Facial. 1999; 4(1): 79-100.

12. Cascaes, S. E. Técnicas ortodônticos para verticalização dos molares inferiores. 1ra 
Edición. Curitiba: Monografía presentada a la Universidad Tuiuti de Paraná para la obtención del título de especialista en ortodoncia; 2014.

13. Roberts, W. W., Chacker, F. M., \& Burstone, C. J. A segmental approach to mandibular molar uprighting. American Journal of Orthodontics. 1982; 81(3): 177-184. 